\title{
Bench-to-bedside and bedside back to the bench; coordinating clinical and experimental traumatic brain injury studies
}

\author{
Denes V. Agoston ${ }^{1,2 *}$, Mårten Risling ${ }^{2}$ and Bo-Michael Bellander ${ }^{3}$ \\ 1 Department of Anatomy, Physiology and Genetics, Uniformed Services University of the Health Sciences, Bethesda, MD, USA \\ 2 Experimental Traumatology, Department of Neuroscience, Karolinska Institutet, Stockholm, Sweden \\ ${ }^{3}$ Department of Clinical Neuroscience, Section for Neurosurgery, Karolinska University Hospital, Solna, Sweden
}

\section{Edited by:}

Mattias Sköld, Uppsala University, Sweden

Reviewed by:

Charmaine Childs, National University of Singapore, Singapore

Fredrik Clausen, Uppsala University, Sweden

\section{*Correspondence:}

Denes V. Agoston, Experimental

Traumatology, Department of

Neuroscience Karolinska Institutet

Stockholm, Sweden.

e-mail:denes.agoston@ki.se
Traumatic brain injury (TBI) is one of the leading cause of death and long-term disability in virtually every country. Advances in neurointensive care have resulted in steadily decreasing morbidity, but the number of individuals with severe long-term disability have not changed significantly and the number of moderate disability has shown steady increase over the last 3 decades. Despite years of intensive preclinical research - and millions spent - there are virtually no drugs specifically developed to mitigate the consequences of TBI. Here we discuss some of the existing gaps between clinical and experimental TBI studies that may have contributed to the current status. We do this hoping that clinical, basic, and translational scientists will design and coordinate studies in order to achieve maximum benefits forTBI patients. In conclusion, we suggest to: (1) Develop consensus-based guidelines for experimental TBI research, similar to "best practices" in the clinic; (2) Generate a consensus-based template for clinical data collection and deposition as well as for experimental TBI data collection and deposition; (3) Use a systems biology approach and create a database for integrating existing data from basic and clinical research.

Keywords: neurotrauma, clinical, experimental, gaps
This is the golden age of traumatic brain injury (TBI) research, veterans of the field have been saying. Increased public awareness, financial support, and novel methodologies have been aligned creating a seemingly perfect environment to find better treatments for TBI patients. We believe that reducing existing incongruities between clinical and experimental TBI research will contribute to saving more lives and significantly improve the quality of life after TBI. Improving the long-term functional outcome is an especially critical issue. Although progress in neurointensive care has resulted in steadily decreasing death rates, the number of individuals with severe disability has not changed significantly. Alarmingly, the rate of long-term, moderate disability after TBI has actually increased over the last 3 decades (Elf et al., 2002).

Here we discuss some of the existing incongruities between clinical and experimental TBI studies. We do this hoping that clinical, basic, and translational scientists will design and coordinate studies in order to achieve maximum benefits for TBI patients. The existing mismatches between clinical and experimental TBI research we discuss below are in (1) the methods, (2) the biosamples, and (3) the timing of "sampling." Some of the differences are dictated by clear technical, methodological, and ethical constraints while others, however, are not. Those that are not can and should be modified to bring the two fields closer together. In order to illustrate the existing differences between clinical and experimental TBI, we have attempted to use numbers to illustrate our points. The differences may be even greater than depicted, since only a fraction of clinical TBI cases are part of clinical studies whereas results from most experimental TBI research are published.

\section{METHODS}

In Table 1 we have listed the methods most frequently used in clinical and experimental TBI studies as well as the type of data output that is commonly generated. In clinical TBI one of the main sources of diagnostic information is in vivo imaging (Maas et al., 1997; Belanger et al., 2007; Coles, 2007; Gallagher et al., 2007; Jagoda et al., 2009). In contrast, there are very few animal studies using similar imaging approaches. We believe that by employing different in vivo imaging modalities in animal studies, experimental, and clinical scientists will be better equipped to cross-validate their respective data and will help interpret their respective findings. In clinical settings, functional information is derived from various injury severity tests (Saatman et al., 2008). These include the Glasgow Coma Scale (GCS), as well as from other neurobehavioral assessments (Maas et al., 1997). Such functional assessments, in various combinations, are routinely used in virtually every clinical setting. While it is not possible to mimic GCS rates which measures alertness (eye opening), long-tract motor signs (extendflex, etc.), and higher-order (verbal) functions, there are basic neurobehavioral tests available for rodents (Crawley, 2003). Although these are inexpensive tests, surprisingly few experimental studies use them routinely. They are extremely valuable to monitor the extent and the temporal changes of functional deficits after experimental injury. Even with the caveat that these tests are highly observer dependent, they can provide critical functional information. Assessing basic neurobehavioral functions after injury would also help to address inter-animal variability, an important issue that frequently arises even during the best-designed animal 
TBI experiments. Neurobehavioral assays are highly sensitive and can reflect potential differences between animals with identical parameters of experimental injury. In combination with additional in vivo cellular and molecular analyses, these tests would be immensely valuable in bridging the gap between experimental and clinical TBI research. In the clinical (and especially neurointensive care) setting, cerebral monitoring is another widely used, vital source of diagnostic information (Maas et al., 1997; Bratton et al., 2007a,b,c,d,e,f,g; Saatman et al., 2008; Sande and West, 2010). It is far less frequently used in experimental TBI, partly due to technical issues such as the sizes of the various probes and the necessary modification of equipment and software. Importantly, the most frequently used animal of TBI - the rodent - is not especially amenable to these techniques. Further sources of important diagnostic information in clinical TBI are the systemic changes in physiology, metabolism, and the inflammatory response (Bratton et al., 2007a,b,c,d,e,f,g; Saatman et al., 2008; Sande and West, 2010). Monitoring some of the same parameters in experimental TBI and analyzing data in the context of histological and molecular outcome measures could be of substantial value in reducing incongruities between clinical and experimental TBI research. Transcranial Doppler (TCD) and quantitative electroencephalography ( $\mathrm{qEEG}$ ) have been used in the clinical setting to provide information about blood flow, seizure activity, ischemia, and vasospasm (Newell and Aaslid, 1992; Wallace et al., 2001; Thakor and Tong, 2004; Nuwer et al., 2005; Tsivgoulis et al., 2009). Animal versions of the methods along with the equipment exist. However, these techniques are infrequently used in experimental TBI research. Again, combining the readouts of TCD and qEEG measurements with biochemical and histological analyses of the brain tissue in experimental TBI could help to validate and interpret clinical TCD and qEEG data. Biochemical assays of blood (serum), cerebrospinal fluid (CSF), and cerebral microdialysate (MD) that identify changes in cerebral metabolism, oxygenation, and glucose metabolism provide clinicians with important data of disease progression (Maas et al., 1997; Bratton et al., 2007a,b,c,d,e,f,g; Saatman et al., 2008; Sande and West, 2010). These data are routinely obtained in clinical TBI (even if data is not necessarily published), but less frequently included in experimental TBI research. Cerebral microdialysis can be used in rodents to obtain interstitial fluid from various regions of the brain. MD samples along with CSF can be then analyzed in a similar way to clinical practice and the obtained data should help in interpreting the clinical findings (Bellander et al., 2004; Hillered et al., 2006; Goodman and Robertson, 2009). Proteomics analysis of blood (serum) and CSF has shown some initial promise in predicting outcome in clinical studies (Jagoda et al., 2008; Saatman et al., 2008; Cadosch et al., 2010). Similar studies have also been performed in experimental TBI (Hayes et al., 2009). With its controlled conditions, experimental TBI can provide an excellent framework for systematic evaluation of markers. In combination with additional functional, cellular, and molecular analyses, such studies would constitute an important step toward validation of the markers and also to an increased understanding of the underlying pathologies.

One of the most frequent "outcome measures" in experimental TBI is obtained by using various histological techniques ranging from classical histopathology to in situ hybridization histochemistry. While clinical neuropathology has been a powerful tool to identify some of the fundamental anatomical and cellular changes associated with TBI, for understandable reasons, it is unable to provide the necessary spatial and temporal resolutions that experimental TBI studies can offer. Combining histological analysis with in vivo imaging, neurobehavioral testing, and biochemical assays would be especially critical in integrating experimental and clinical data. The power and resolution of the various "omics" e.g., genomics, transcriptomics (Di Pietro et al., 2010), lipidomics (Sparvero et al., 2010), and last but not least proteomics - have started to generate significant new data in clinical and experimental TBI research (Oli et al., 2009; Ottens et al., 2010). The flexibility in designing experimental TBI studies that can mimic clinical scenarios would make such studies even more valuable, especially if they are combined with additional functional/neurobehavioral and morphological/histological "outcome measures."

\section{BIOSAMPLES}

In Table 2, we listed the main types of biosamples collected for the various assays in clinical vs. experimental TBI studies. In clinical settings, blood (systemic, arterial, and venous) is collected and analyzed routinely for diagnostic purposes (yet not necessarily published). Similar analysis is far less frequently performed in experimental TBI studies. However, blood could easily be collected from experimental animals at all time points required to match clinical sampling times. Performing some of the same biochemical, cellular, and metabolic analyses of experimental samples could provide information that can be directly compared with clinical data. CSF has long been used for monitoring progression and outcome in clinical TBI (Saatman et al., 2008; Kochanek et al., 2010). Admittedly, it can be challenging to obtain sufficient quantity of CSF from the rat, especially at multiple time points. However, such sampling and the subsequent analysis of CSF samples could be very useful in enabling direct comparison of clinical and experimental data especially in the context of outcome measures including neurobehavioral, cellular, and molecular as discussed above. Cerebral MD studies have been performed in rat models of TBI as well as in clinical TBI studies (Maurer, 2010; O'Connor et al., 2011). While there are issues with cerebral MD, it can be a unique source of information about both spatially and temporally restricted pathological changes at the molecular level (Saatman et al., 2008).

\section{TIMING}

In Table 3 we have listed the typical time points at which biosamples are obtained during clinical vs. experimental TBI studies. A significant amount of clinical data is collected during the early time periods (hours and days) of the post-injury phase using imaging, neurobehavioral testing, cerebral monitoring, and biochemical assays. In addition to the other differences (see Table 1), such early time points are not usually monitored in experimental TBI. It should be noted that one must also consider the significant differences between humans and rodents with respect to metabolic rate, life span, etc., when attempting to match clinical and experimental time points (Quinn, 2005).

The strengths and possibilities associated with the design of experimental studies include good control of exposure data 
Table 1 | Clinical vs. experimental research: a comparison of methods.

\begin{tabular}{|c|c|c|c|c|c|c|}
\hline \multirow[t]{2}{*}{ Methods } & \multicolumn{2}{|c|}{ Use* } & \multicolumn{4}{|c|}{ Type of information } \\
\hline & Clinical & Experimental & Temporal & Spatial & Molecular & Functional \\
\hline \multicolumn{7}{|l|}{ IN VIVO IMAGING } \\
\hline $\mathrm{CT}$ & 2685 & 150 & Yes & Yes & No & No \\
\hline $\mathrm{MRI}$ & 3595 & 435 & Yes & Yes & Yes/no & Yes \\
\hline PET & 230 & 37 & Yes & Yes & Yes & Yes/no \\
\hline \multicolumn{7}{|c|}{ NEUROBEHAVIORAL ASSAYS } \\
\hline Injury severity & 4983 & 705 & Yes & No & No & Yes \\
\hline Neurobehavioral & 289 & 140 & Yes & No & No & Yes \\
\hline \multicolumn{7}{|l|}{ CEREBRAL MONITORING } \\
\hline $\mathrm{ICP}$ & 1089 & 220 & Yes & No & No & Yes \\
\hline CBF & 1862 & 879 & Yes & No & No & Yes \\
\hline TCD & 301 & 19 & Yes & No & No & Yes \\
\hline qEEG & 2258 & 375 & Yes & Yes/no & No & Yes \\
\hline \multicolumn{7}{|l|}{ BIOCHEMICAL ASSAYS } \\
\hline Blood/metabolites & 46 & 42 & Yes & No & Yes & No \\
\hline CSF/metabolites & 25 & 5 & Yes & No & Yes & No \\
\hline MD/metabolites & 20 & 15 & Yes & Yes & Yes & No \\
\hline Blood/protein biomarkers & 291 & 203 & Yes & No & Yes & No \\
\hline CSF/protein biomarkers & 91 & 30 & Yes & No & Yes & No \\
\hline MD/protein biomarkers & 6 & 1 & Yes & Yes & Yes & No \\
\hline \multicolumn{7}{|c|}{ IN VITRO ANALYSIS OF BRAIN TISSUE } \\
\hline Histopathology & 7461 & 6005 & No & Yes & No & Yes/no \\
\hline $\mathrm{IHC}$ & 434 & 1862 & No & Yes & Yes & Yes/no \\
\hline ISHH & 36 & 392 & No & Yes & Yes & Yes/no \\
\hline EM & 144 & 509 & No & Yes & Yes/no & Yes/no \\
\hline Genomics & 45 & 47 & No & Yes & Yes & Yes/no \\
\hline Proteomics & 42 & 43 & No & Yes & Yes & Yes/no \\
\hline
\end{tabular}

*Use is defined as relative frequency of use based on the number of publications retrieved by searching PubMed using the search terms: "traumatic brain injury" in combination with the methods presented in the left column together with the limitation "animal" or "human." The number of papers found is registered in the second and third columns on the left. CT, computer tomography; MRI, magnetic resonance imaging; PET, positron emission tomography; ICP, intracranial pressure; CBF, cerebral blood flow; TCD, transcranial Doppler; qEEG, quantitative electroencephalography; CSF, cerebrospinal fluid; MD, microdialysate; IHC, immunohistochemistry; ISHH, in situ hybridization histochemistry; EM, electron microscopy.

Table 2 | Comparing biosamples collected for the various analyses in clinical and in experimental TBI studies.

\begin{tabular}{llcrll}
\hline Samples & Blood & Serum & CSF & Microdialysate & Brain tissue \\
\hline Experimental & 14 & 0 & 375 & 173 & 4264 \\
Clinical & 96 & 24 & 1691 & 225 & 3636
\end{tabular}

Search on Entrez PubMed using "traumatic brain injury" in combination with the parameters presented in the left column together with the limitation "animal" or "human." The number of papers found is registered.

(the physics of the injury). Ongoing tests with helmet-mounted accelerometers can provide more detailed exposure data (Rigby et al., 2011) helping to validate predictions from experimental studies. FEM (finite element modeling) can be used as a tool to calculate force distribution in both clinical and experimental TBI, but this approach needs to be validated by actual detailed biological findings and physics data that usually would not be available in clinical materials.

Age as well as the genetic background can be selected with different rodent strains, gene knock-out models, etc. Obviously, this can be challenging to realistically mimic in clinical TBI studies. It is also challenging to find counterparts to complex data on population backgrounds, such as single-nucleotide polymorphisms for important genes that are available in clinical materials. For numerous reasons, experimental TBI research rarely generates truly longitudinal data. A few clinical databanks such as the Vietnam Head Injury study provide exceptionally long follow-ups (extending for more than 3 decades), imaging data, as well as detailed genetic and cognitive data in combination with basic preinjury data (Raymont et al., 2010). Such materials are superbly suitable to bridge the gap between clinical and experimental studies. In summary, we feel that is exceedingly important to find the connecting points that could help us take full advantage of the different strengths in clinical and experimental materials. 
Table 3 | Comparing the temporal pattern of performing the various analyses in clinical and in experimental TBI studies.

\begin{tabular}{|c|c|c|c|c|}
\hline \multirow[t]{2}{*}{ Methods } & \multicolumn{4}{|c|}{ Time } \\
\hline & Hours & Days & Weeks & Months \\
\hline \multicolumn{5}{|l|}{ In vivo imaging } \\
\hline Clinical & +++ & ++ & + & \pm \\
\hline Experimental & \pm & + & \pm & - \\
\hline \multicolumn{5}{|l|}{ Neurobehavioral } \\
\hline Clinical & +++ & +++ & +++ & +++ \\
\hline Experimental & \pm & \pm & ++ & \pm \\
\hline \multicolumn{5}{|c|}{ Cerebral monitoring } \\
\hline Clinical & +++ & ++ & - & - \\
\hline Experimental & - & - & - & - \\
\hline \multicolumn{5}{|c|}{ Biochemical assays } \\
\hline Clinical & +++ & +++ & + & \pm \\
\hline Experimental & - & \pm & \pm & - \\
\hline \multicolumn{5}{|c|}{ In vitro analysis of brain tissue } \\
\hline Clinical & \pm & \pm & \pm & \pm \\
\hline Experimental & +++ & ++ & + & - \\
\hline
\end{tabular}

Typical frequency of use: not typically used; \pm , rarely used; +, typically used; ++, frequently used; +++ , routinely used. The indications are based on outputs from literature searches and personal communications.

We would like to close this article by suggesting a few easily implementable solutions that could help coordinate clinical and experimental TBI studies: (1) Develop consensus-based guidelines for experimental TBI research, similar to "best practices" in the

\section{REFERENCES}

Belanger, H. G., Vanderploeg, R. D., Curtiss, G., and Warden, D. L. (2007). Recent neuroimaging techniques in mild traumatic brain injury. J. Neuropsychiatry Clin. Neurosci. 19, 5-20.

Bellander, B. M., Cantais, E., Enblad, P., Hutchinson, P., Nordstrom, C. H., Robertson, C., Sahuquillo, J., Smith, M., Stocchetti, N., Ungerstedt, U., Unterberg, A., and Olsen, N. V. (2004). Consensus meeting on microdialysis in neurointensive care. Intensive Care Med. 30, 2166-2169.

Bratton, S. L., Chestnut, R. M., Ghajar, J., Mcconnell Hammond, F. F., Harris, O. A., Hartl, R., Manley, G. T., Nemecek, A., Newell, D. W., Rosenthal, G., Schouten, J., Shutter, L., Timmons, S. D., Ullman, J. S., Videtta, W., Wilberger, J. E., and Wright, D. W. (2007a). Guidelines for the management of severe traumatic brain injury. I. Blood pressure and oxygenation. J. Neurotrauma 24(Suppl. 1), S7-S13.

Bratton, S. L., Chestnut, R. M., Ghajar, J., Mcconnell Hammond, F. F., Harris, O. A., Hartl, R., Manley, G. T., Nemecek, A., Newell, D. W.,
Rosenthal, G., Schouten, J., Shutter, L., Timmons, S. D., Ullman, J. S., Videtta, W., Wilberger, J. E., and Wright, D. W. (2007b). Guidelines for the management of severe traumatic brain injury. IX. Cerebral perfusion thresholds. J. Neurotrauma 24(Suppl. 1), S59-S64.

Bratton, S. L., Chestnut, R. M., Ghajar, J., Mcconnell Hammond, F. F. Harris, O. A., Hartl, R., Manley, G. T., Nemecek, A., Newell, D. W., Rosenthal, G., Schouten, J., Shutter, L., Timmons, S. D., Ullman, J. S., Videtta, W., Wilberger, J. E., and Wright, D. W. (2007c). Guidelines for the management of severe traumatic brain injury. VI. Indications for intracranial pressure monitoring. J. Neurotrauma 24(Suppl. 1), S37-S44.

Bratton, S. L., Chestnut, R. M., Ghajar, J., Mcconnell Hammond, F. F., Harris, O. A., Hartl, R., Manley, G. T., Nemecek, A., Newell, D. W., Rosenthal, G., Schouten, J., Shutter, L., Timmons, S. D., Ullman, J. S., Videtta, W., Wilberger, J. E., and Wright, D. W. (2007d). Guidelines for the management of severe traumatic brain injury. VII. Intracranial

clinic. This would require researchers to design, analyze, and report their experiments according to accepted "best practices." These "best practices" should mimic clinical scenarios and include neurobehavioral tests, assays for monitoring systemic changes in the peripheral blood at time points mirroring clinical sampling times, and so on. (2) Generate a consensus-based template for both clinical AND experimental TBI data collection and deposition. Finally, (3) use a systems biology approach and establish a database into which current data from both clinical and experimental TBI research can be imported and analyzed in the context of existing neurobiology knowledge. This would greatly help to organize and utilize the treasure of existing data derived from past clinical and experimental TBI studies. There are different potential organizational principles (like functional neuroanatomy) that could provide the backbone for such a database. Such a database should be interfaced with cellular, molecular, and disease databases already in existence. One example of a disease-oriented database which collects and disseminates relevant information is the international collaborative effort of the Biomedical Informatics for the Management of Cerebral Aneurysms (@neurIST).

We believe that by implementing some of these measures, clinical and experimental TBI research will become more relevant, effectual, and would ultimately help to make the next decade truly a golden age of TBI research. We would like to open this paper for discussion. Your views, comments, etc., are very welcome.

\section{ACKNOWLEDGMENTS}

We thank Drs. Mark Mahan and Mattias Sköld for discussing the manuscript and Mrs. Victoria Hammarstedt for editorial assistance.

pressure monitoring technology. $J$. Neurotrauma 24(Suppl. 1), S45-S54.

Bratton, S. L., Chestnut, R. M., Ghajar, J., Mcconnell Hammond, F. F., Harris, O. A., Hartl, R., Manley, G. T., Nemecek, A., Newell, D. W., Rosenthal, G., Schouten, J., Shutter, L., Timmons, S. D., Ullman, J. S., Videtta, W., Wilberger, J. E., and Wright, D. W. (2007e) Guidelines for the management of severe traumatic brain injury. VIII. Intracranial pressure thresholds. J. Neurotrauma 24(Suppl. 1), S55-S58.

Bratton, S. L., Chestnut, R. M., Ghajar, J., Mcconnell Hammond, F. F., Harris, O. A., Hartl, R., Manley, G. T., Nemecek, A., Newell, D. W., Rosenthal, G., Schouten, J., Shutter, L., Timmons, S. D., Ullman, J. S., Videtta, W., Wilberger, J. E., and Wright, D. W. (2007f). Guidelines for the management of severe traumatic brain injury. X. Brain oxygen monitoring and thresholds. J. Neurotrauma 24(Suppl. 1), S65-S70.

Bratton, S. L., Chestnut, R. M., Ghajar, J., Mcconnell Hammond, F. F., Harris, O. A., Hartl, R., Manley, G. T., Nemecek, A., Newell, D. W.,
Rosenthal, G., Schouten, J., Shutter, L., Timmons, S. D., Ullman, J. S., Videtta, W., Wilberger, J. E., and Wright, D. W. (2007g). Guidelines for the management of severe traumatic brain injury. XIV. Hyperventilation. J. Neurotrauma 24(Suppl. 1), S87-S90.

Cadosch, D., Thyer, M., Gautschi, O. P., Lochnit, G., Frey, S. P., Zellweger, R., Filgueira, L., and Skirving, A. P. (2010). Functional and proteomic analysis of serum and cerebrospinal fluid derived from patients with traumatic brain injury: a pilot study. ANZ J. Surg. 80, 542-547.

Coles, J. P. (2007). Imaging after brain injury. Br. J. Anaesth. 99, 49-60.

Crawley, J. N. (2003). Behavioral phenotyping of rodents. Comp. Med. 53, 140-146.

Di Pietro, V., Amin, D., Pernagallo, S., Lazzarino, G., Tavazzi, B., Vagnozzi R., Pringle, A., and Belli, A. (2010). Transcriptomics of traumatic brain injury: gene expression and molecular pathways of different grades of insult in a rat organotypic hippocampal culture model. J. Neurotrauma 27, 349-359. 
Elf, K., Nilsson, P., and Enblad, P. (2002). Outcome after traumatic brain injury improved by an organized secondary insult program and standardized neurointensive care. Crit. Care Med. 30, 2129-2134.

Gallagher, C. N., Hutchinson, P. J., and Pickard, J. D. (2007). Neuroimaging in trauma. Curr. Opin. Neurol. 20, 403-409.

Goodman, J. C., and Robertson, C. S. (2009). Microdialysis: is it ready for prime time? Curr. Opin. Crit. Care 15, 110-117.

Hayes, R. L., Robinson, G., Muller, U., and Wang, K. K. (2009). Translation of neurological biomarkers to clinically relevant platforms. Methods Mol. Biol. 566, 303-313.

Hillered, L., Persson, L., Nilsson, P., Ronne-Engstrom, E., and Enblad, P. (2006). Continuous monitoring of cerebral metabolism in traumatic brain injury: a focus on cerebral microdialysis. Curr. Opin. Crit. Care 12, 112-118.

Jagoda, A. S., Bazarian, J. J., Bruns, J. J. Jr., Cantrill, S. V., Gean, A. D., Howard, P. K., Ghajar, J., Riggio, S., Wright, D. W., Wears, R. L., Bakshy, A., Burgess, P., Wald, M. M., and Whitson, R. R. (2008). Clinical policy: neuroimaging and decision making in adult mild traumatic brain injury in the acute setting. Ann. Emerg. Med. 52, 714-748.

Jagoda, A. S., Bazarian, J. J., Bruns, J. J. Jr., Cantrill, S. V., Gean, A. D., Howard, P. K., Ghajar, J., Riggio, S., Wright, D. W., Wears, R. L., Bakshy, A., Burgess, P., Wald, M. M., and Whitson, R. R. (2009). Clinical policy: neuroimaging and decision making in adult mild traumatic brain injury in the acute setting. J. Emerg. Nurs. 35, e5-e40.

Kochanek, P. M., Bell, M. J., and Bayir, H. (2010). Quo vadis 2010? - carpe diem: challenges and opportunities in pediatric traumatic brain injury. Dev. Neurosci. 32, 335-342.

Maas, A. I., Dearden, M., Teasdale, G. M., Braakman, R., Cohadon, F., Iannotti, F., Karimi, A., Lapierre, F., Murray, G., Ohman, J., Persson, L., Servadei, F., Stocchetti, N., and Unterberg, A. (1997). EBICguidelines for management of severe head injury in adults. European Brain Injury Consortium. Acta Neurochir. (Wien) 139, 286-294.

Maurer, M. H. (2010). Proteomics of brain extracellular fluid (ECF) and cerebrospinal fluid (CSF). Mass Spectrom. Rev. 29, 17-28.

Newell, D. W., and Aaslid, R. (1992). Transcranial Doppler: clinical and experimental uses. Cerebrovasc. Brain Metab. Rev. 4, 122-143.

Nuwer, M. R., Hovda, D. A., Schrader, L. M., and Vespa, P. M. (2005). Routine and quantitative EEG in mild traumatic brain injury. Clin. Neurophysiol. 116, 2001-2025.

O'Connor, W. T., Smyth, A., and Gilchrist, M. D. (2011). Animal models of traumatic brain injury: a critical evaluation. Pharmacol. Ther. 130, 106-113.

Oli, M. W., Hayes, R. L., Robinson, G., and Wang, K. K. (2009). Traumatic brain injury biomarkers: from pipeline to diagnostic assay development. Methods Mol. Biol. 566, 293-302.
Ottens, A. K., Bustamante, L., Golden, E. C., Yao, C., Hayes, R. L., Wang, K. K., Tortella, F. C., and Dave, J. R. (2010). Neuroproteomics: a biochemical means to discriminate the extent and modality of brain injury. J. Neurotrauma 27, 1837-1852.

Quinn, R. (2005). Comparing rat's to human's age: how old is my rat in people years? Nutrition 21, 775-777.

Raymont, V., Salazar, A. M., Lipsky, R. Goldman, D., Tasick, G., and Grafman, J. (2010). Correlates of posttraumatic epilepsy 35 years following combat brain injury. Neurology 75, 224-229.

Rigby, P., Wong, J., Juhas, B., Eslami, P. Rapo, M., and Baumer, T. (2011). "Using helmet sensors in predicting head kinematics," in A Survey of Blast Injury across the Full Landscape of Military Science: NATO.

Saatman, K. E., Duhaime, A. C., Bullock, R., Maas, A. I., Valadka, A., and Manley, G. T. (2008). Classification of traumatic brain injury for targeted therapies. J. Neurotrauma 25, 719-738.

Sande, A., and West, C. (2010). Traumatic brain injury: a review of pathophysiology and management. J. Vet. Emerg. Crit. Care (San Antonio) 20, 177-190

Sparvero, L. J., Amoscato, A. A., Kochanek, P. M., Pitt, B. R., Kagan, V. E., and Bayir, H. (2010). Mass-spectrometry based oxidative lipidomics and lipid imaging: applications in traumatic brain injury. $J$. Neurochem. 115, 1322-1336.

Thakor, N. V., and Tong, S. (2004). Advances in quantitative electroencephalogram analysis methods. Annu. Rev. Biomed. Eng. 6 , 453-495.

Tsivgoulis, G., Alexandrov, A. V., and Sloan, M. A. (2009). Advances in transcranial Doppler ultrasonography. Curr. Neurol. Neurosci. Rep. 9, 46-54.

Wallace, B. E., Wagner, A. K., Wagner, E. P., and Mcdeavitt, J. T. (2001) A history and review of quantitative electroencephalography in traumatic brain injury. J. Head Trauma Rehabil. 16, 165-190.

Conflict of Interest Statement: The authors declare that the research was conducted in the absence of any commercial or financial relationships that could be construed as a potential conflict of interest.

Received: 18 October 2011; paper pending published: 25 November 2011; accepted: 03 January 2012; published online: 02 February 2012.

Citation: Agoston DV, Risling $M$ and Bellander B-M (2012) Bench-to-bedside and bedside back to the bench; coordinating clinical and experimental traumatic brain injury studies. Front. Neur. 3:3. doi: 10.3389/fneur.2012.00003

This article was submitted to Frontiers in Neurotrauma, a specialty of Frontiers in Neurology.

Copyright (c) 2012 Agoston, Risling and Bellander. This is an open-access article distributed under the terms of the Creative Commons Attribution Non Commercial License, which permits noncommercial use, distribution, and reproduction in other forums, provided the original authors and source are credited. 